\title{
A new model for QPOs in accreting black holes: application to the microquasar GRS $1915+105$
}

\author{
J. Pétri
}

Received: 21 May 2008 / Accepted: 15 September 2008 / Published online: 3 October 2008

(C) The Author(s) 2008. This article is published with open access at Springerlink.com

\begin{abstract}
In this paper we extend the idea suggested previously by Pétri (Astron. Astrophys. 439:L27, 2005a; 443:777, 2005b) (papers I and II) that the high frequency quasiperiodic oscillations (HF-QPOs) observed in low-mass Xray binaries (LMXBs) may be explained as a resonant oscillation of the accretion disk with a rotating asymmetric background (gravitational or magnetic) field imposed by the compact object. Here, we apply this general idea to black hole binaries. It is assumed that a test particle experiences a similar parametric resonance mechanism such as the one described in paper I and II but now the resonance is induced by the interaction between a spiral density wave in the accretion disk, excited close to the innermost stable circular orbit, and vertical epicyclic oscillations. We use the Kerr spacetime geometry to deduce the characteristic frequencies of this test particle. The response of the test particle is maximal when the frequency ratio of the two strongest resonances is equal to 3:2 as observed in black hole candidates. Finally, applying our model to the microquasar GRS $1915+105$, we reproduce the correct value of several HFQPOs. Indeed the presence of the 168/113/56/42/28 Hz features in the power spectrum time analysis is predicted. Moreover, based only on the two HF-QPO frequencies, our
\end{abstract}

\section{J. Pétri ( $\square)$}

Observatoire Astronomique de Strasbourg,

11, rue de l'Université, 67000 Strasbourg, France

e-mail: petri@astro.u-strasbg.fr

\section{J. Pétri}

Centre d'étude des Environnements Terrestre et Planétaires,

10-12 avenue de l'Europe, 78140 Vélizy, France

J. Pétri

Laboratoire de Radio-Astronomie, École Normale Supérieure,

24 rue Lhomond, 75005 Paris, France model is able to constrain the mass $M_{\mathrm{BH}}$ and angular momentum $a_{\mathrm{BH}}$ of the accreting black hole. We show the relation between $M_{\mathrm{BH}}$ and $a_{\mathrm{BH}}$ for several black hole binaries. For instance, assuming a black hole weakly or mildly rotating, i.e. $a_{\mathrm{BH}} \leq 0.5 G M_{\mathrm{BH}} / c^{2}$, we find that for GRS $1915+105$ its mass satisfies $13 M_{\odot} \leq M_{\mathrm{BH}} \leq 20 M_{\odot}$. The same model applied to two other well-known BHCs gives for GRO J1655-40 a mass $5 M_{\odot} \leq M_{\mathrm{BH}} \leq 7 M_{\odot}$ and for XTE $\mathrm{J} 1550-564$ a mass $8 M_{\odot} \leq M_{\mathrm{BH}} \leq 11 M_{\odot}$. This is consistent with other independent estimations of the black hole mass. Finally for H1743-322, we found the following bounds, $9 M_{\odot} \leq M_{\mathrm{BH}} \leq 13 M_{\odot}$.

Keywords Accretion, accretion disks - Black hole physics · Methods: analytical $\cdot$ Relativity $\cdot$ X-rays: binaries

\section{Introduction}

High frequency quasi-periodic oscillations (HF-QPOs) are common features to all accreting compact objects, be they neutron stars, white dwarfs or black holes. A number of recent observations have revealed the existence of these HFQPOs in several black hole binaries (Strohmayer 2001a; McClintock and Remillard 2003; Remillard and McClintock 2006). Usually, a pair of HF-QPOs appears in a 3:2 ratio. If these oscillations are connected to the orbital motion of the accretion disk at its inner edge as predicted by several models, these QPOs become a useful test of gravity in the strong field regime.

The 3:2 ratio was first noticed by Abramowicz and Kluźniak (2001). In order to explain this ratio, they introduced a resonance mechanism between orbital and epicyclic motion around Kerr black holes therefore leading to an estimate of their mass and spin. Kluźniak et al. (2004) showed 
that the twin $\mathrm{kHz}-\mathrm{QPO}$ is explained by a non linear resonance in the epicyclic motion of the accretion disk. Rebusco (2004) developed the analytical treatment of these oscillations. This parametric epicyclic resonance model of hydrodynamical modes in the accretion disk was applied by Kluźniak and Abramowicz (2002) to some microquasars. They pointed out the 3:2 ratio in the HF-QPOs observed in GRO J1655-40, XTE J1550-564 and GRS $1915+105$. Moreover, in this latter black hole binary, Strohmayer (2001b) reported another pair of frequencies, namely $69.2 \mathrm{~Hz}$ and $41.5 \mathrm{~Hz}$, which are in a 5:3 ratio as noticed by Kluźniak and Abramowicz (2002) and supports the parametric resonance model. Furthermore, Rezzolla et al. (2003) suggested that the HF-QPOs in black hole binaries are related to p-mode oscillation in a non Keplerian torus.

Nevertheless, the propagation of the emitted photons in curved spacetime can also produce some intrinsic peaks in the Fourier spectrum of the light curves (Schnittman and Bertschinger 2004). In combination with a vertically oscillating torus, the gravitational lensing effect can also reproduce the 3:2 ratio (Bursa et al. 2004; Schnittman and Rezzolla 2005).

Resonances in the geodesic motion of a single particle have been investigated by Abramowicz et al. (2003). The specific coupling force between radial and vertical oscillation was left unspecified. Their results for non-linear resonance were applied to accreting neutron stars.

In this paper, we describe a coupling between spiral density waves in the accretion disk and epicyclic motions of test particles. It is divided in two parts. In Sect. 2 we specify the perturbation pattern used in our model. Then the equation of motions due to the perturbation are derived leading to some resonance conditions. In Sect. 3, the results are applied to GRS $1915+105$ for which several components in the Fourier time analysis are predicted in agreement with the HF-QPOs detected for this object. We also put some constrain on the mass-spin relation for several BHCs.

\section{The model}

In this section, we describe the main features of the model, starting with a simple treatment of the accretion disk, assumed to be made of non interacting single particles orbiting in the equatorial plane of the black hole as was already done in the previous studies (see Pétri 2005a, 2005b). We again neglect the hydrodynamical aspects of the disk such as pressure. However, a detailed (magneto-)hydrodynamical treatment of the response of the disk to gravitational or magnetic perturbations has been given in Pétri $(2005$ c, 2006). For a first approach to the problem, we neglect this refinement. Particles evolve in the unperturbed stationary background gravitational field of the black hole. In this sim- plistic approach, we want to point out the effect of a spiral density wave propagating radially outwards in the accretion disk. In order to account for general-relativistic effects, we use the Kerr metric to perform our calculations. We will show that a local outgoing one-armed m-mode will induce a beat with the test particles and excite vertical oscillations parametrically. Indeed, Mao et al. (2008) showed that oscillatory modes are excited close to the innermost stable circular orbit (ISCO) and propagate outwards to several tens of Schwarzschild radii with constant frequency (nearly equal to the maximum radial epicyclic frequency) and without attenuation. They also found that these oscillations are locally super-Keplerian. Nevertheless, they studied only axisymmetric modes in a pseudo-Newtonian gravitational field. A more detailed study including full general relativity and asymmetric modes propagation would help to give precise quantitative results. In this paper, we will assume that such asymmetric modes exist and exhibit the same properties as the modes found in Mao et al. (2008).

\subsection{Equation of motion for a test particle}

We use the same procedure as the one described in paper I. However, in order to take into account the spiral structure of the density wave of azimuthal mode number $m$ and frequency $\Omega_{\mathrm{w}}$, we have to change the equation of motion (7) in Pétri (2005a) by modifying the terms " $\cos \left(m\left(\Omega-\Omega_{\mathrm{w}}\right) t\right)$ " in such a way that the spatio-temporal dependency of the argument of "cos" implies a propagation of a pattern of azimuthal mode $m$ at the sound speed $c_{\mathrm{s}}$. We remember that $\Omega$ is the local orbital frequency in the accretion disk. Therefore, in cylindrical coordinates $(r, \varphi, z)$, we choose a phase dependency like $\psi=m \varphi-\Omega_{\mathrm{w}}\left(t-r / c_{\mathrm{s}}\right)$, where $t$ is the time. For a particle in circular orbit at a frequency $\Omega$, we have

$\psi=m \Omega t-\Omega_{\mathrm{w}}\left(t-r / c_{\mathrm{s}}\right)$

This means that viewed from the orbiting particle frame of reference, the perturbation is rotating at the speed $\Omega_{p}=$ $\left|m \Omega-\Omega_{\mathrm{W}}\right|$. We find again a harmonic oscillator with a periodic variation in the eigenfrequency of the system, driven by an external periodic force generated by the perturbation. The modulation being sinusoidal, the Hill equation specializes to the Mathieu equation, a well known ordinary differential equation extensively studied in mathematical physics (Morse and Feshbach 1953).

\subsection{Resonance conditions}

The rotation of the asymmetric pattern induces a sinusoidal variation of the vertical epicyclic frequency $\kappa_{\mathrm{z}}$ leading to the well known Mathieu equation for a given azimuthal mode $m$. To take into account general-relativistic effects, we 
use the characteristic orbital and epicyclic frequencies for the Kerr spacetime geometry, namely

- the orbital frequency

$$
\Omega=\frac{\sqrt{G M_{\mathrm{BH}}}}{r^{3 / 2}+a_{\mathrm{BH}} \sqrt{R_{g}}}=\frac{c^{3}}{G M_{\mathrm{BH}}} \frac{1}{\tilde{r}^{3 / 2}+\tilde{a}}
$$

- the radial epicyclic frequency

$$
\kappa_{\mathrm{r}}=\Omega \sqrt{1-\frac{6}{\tilde{r}}+8 \frac{\tilde{a}}{\tilde{r}^{3 / 2}}-3 \frac{\tilde{a}^{2}}{\tilde{r}^{2}}}
$$

- the vertical epicyclic frequency

$$
\kappa_{\mathrm{Z}}=\Omega \sqrt{1-4 \frac{\tilde{a}}{\tilde{r}^{3 / 2}}+3 \frac{\tilde{a}^{2}}{\tilde{r}^{2}}} .
$$

We introduced the mass of the black hole $M_{\mathrm{BH}}$, its gravitational radius $R_{g}=G M_{\mathrm{BH}} / c^{2}$, the adimensionalized radius and angular momentum, respectively $\tilde{r}=r / R_{g}$ and $\tilde{a}=a_{\mathrm{BH}} / R_{g}$. We expect a parametric resonance related to this time-varying vertical epicyclic frequency. The resonance condition derived from the vertical equation of motion is

$\left|m \Omega-\Omega_{\mathrm{w}}\right|=2 \frac{\kappa_{\mathrm{z}}}{n}$

where $n \geq 1$ is a natural integer. Note that the speed of propagation $c_{\mathrm{S}}$ in the term " $\left(t-r / c_{\mathrm{S}}\right)$ " drops out from the above resonance condition because it is a local description at fixed radius $r$.

In the non-rotating black hole case, $\tilde{a}=0$, we have $\kappa_{\mathrm{z}}=\Omega$. Therefore the parametric resonance conditions (2) splits into two cases, depending on the absolute sign

$\Omega=\frac{n}{m n \pm 2} \Omega_{\mathrm{w}}$

It differs by a factor $1 / \mathrm{m}$ from the resonance condition derived in paper I and II. This discrepancy comes from the choice of the phase dependency here being $\psi$ and describing a radially outward propagating pattern. There are some other resonance mechanisms happening in the accretion disk which are not of interest in the present study. Nevertheless we remind them for completeness. First, forced oscillations occur whenever the driven frequency is equal to the free oscillation frequency

$\left|m \Omega-\Omega_{\mathrm{w}}\right|=\kappa_{\mathrm{z}}$

They are a special case of the above mentioned parametric resonance, corresponding to $n=2$. Second, for the corotation resonance to happen, we should have

$\Omega=\Omega_{\mathrm{w}}$

As a consequence, we find again the same resonance mechanisms as in paper I and II. Equation (3) is also a good approximation for slowly rotating black holes $(\tilde{a} \ll 1)$ because in this case $\kappa_{\mathrm{z}} \approx \Omega$.

We now discuss in detail the case $\tilde{a}=0$ because it is analytically tractable and gives good insight on the resonance frequency even in the fast rotating case. Moreover,
Table 1 Ratio of orbital frequency to the perturbation angular pattern speed at the radii where the parametric resonance is expected to occur.

\begin{tabular}{|c|c|c|c|c|c|c|c|c|}
\hline \multirow[t]{3}{*}{$m$} & \multicolumn{8}{|c|}{$\Omega / \Omega_{\mathrm{w}}$} \\
\hline & \multicolumn{4}{|c|}{$n(-)$} & \multicolumn{4}{|c|}{$n(+)$} \\
\hline & 1 & 2 & 3 & 4 & 1 & 2 & 3 & 4 \\
\hline 1 & -1 & - & 3 & 2 & $1 / 3$ & $1 / 2$ & $3 / 5$ & $2 / 3$ \\
\hline 2 & - & 1 & $3 / 4$ & $2 / 3$ & $1 / 4$ & $1 / 3$ & $3 / 8$ & $2 / 5$ \\
\hline 3 & 1 & $1 / 2$ & $3 / 7$ & $2 / 5$ & $1 / 5$ & $1 / 4$ & $3 / 11$ & $2 / 7$ \\
\hline
\end{tabular}
Left part of the table corresponds to the minus sign whereas the right part corresponds to the plus sign taken in (3)

we checked that in case of a maximally rotating Kerr black hole, $\tilde{a}=1$, the frequencies remains nearly the same as in the case $\tilde{a}=0$ because the resonances occur in regions far away from the ISCO, such that in these regions of the accretion disk, $\kappa_{\mathrm{z}} \approx \Omega$ whatever $\tilde{a}$.

The predicted QPO frequency ratio $\Omega / \Omega_{\mathrm{w}}$ is shown in Table 1 for the first three azimuthal numbers $m=1,2,3$ and the first four integers $n$. The highest orbital frequencies where resonance occurs correspond to $m=1$ and for the minus sign in Table 1 . They are equal to $3 \Omega_{\mathrm{w}}$ and $2 \Omega_{\mathrm{w}}$. Therefore the ratio of the strongest oscillations are in the ratio 3:2 as observed in several black hole binaries. This particular ratio is not a direct consequence of the motion in general relativity but rather an intrinsic property of the parametric resonance. General relativity is only needed in order to describe this resonance mechanism correctly in the strong gravity regime. The third strongest resonance occurs when $\Omega=\Omega_{\mathrm{W}}$ overlapping the corotation resonance. However, if the low azimuthal numbers possess the strongest amplitude in the perturbation power spectrum, the $m=2$ and $m=3$ resonances are expected to be weaker. The most significant QPO frequencies are therefore ordered in the series 3:2:1, the " 1 " only appearing as a weak feature compared to the 3:2 HF-QPOs pair. A quantitative application of the model follows in the next section.

\section{Discussion}

\subsection{Application to GRS $1915+105$}

We test our model with the well studied black hole GRS $1915+105$ for which numerous observations of QPO features are available. Several types of QPOs can be identified in this binary system; low frequency QPOs (LF-QPOs) ranging roughly from 1 to $10 \mathrm{~Hz}$, HF-QPOs for frequencies larger than roughly $70 \mathrm{~Hz}$ and very low frequency QPOs with frequencies less than $1 \mathrm{~Hz}$ (Fender and Belloni 2004). HF-QPO frequencies are detected at $v_{1}=113 \mathrm{~Hz}$ and $v_{2}=165 \mathrm{~Hz}$ (Remillard and McClintock 2006). Other LFQPOs have also been detected for this object. For instance, 
Table 2 QPO frequencies predicted for GRS 1915+ 105 from the orbital motion where parametric resonance occurs. The most interesting frequencies are marked in bold face

\begin{tabular}{|c|c|c|c|c|c|c|c|c|}
\hline \multirow[t]{3}{*}{$m$} & \multicolumn{8}{|c|}{$v_{\mathrm{QPO}}($ in $\mathrm{Hz}$ ) for GRS $1915+105$} \\
\hline & \multicolumn{4}{|l|}{$n(-)$} & \multicolumn{4}{|c|}{$n(+)$} \\
\hline & 1 & 2 & 3 & 4 & 1 & 2 & 3 & 4 \\
\hline 1 & -56.0 & - & 168.0 & 112.0 & 18.7 & 28.0 & 33.6 & 37.3 \\
\hline 2 & - & 56.0 & 42.0 & 37.3 & 14.0 & 18.7 & 21.0 & 22.4 \\
\hline 3 & 56.0 & 28.0 & 24.0 & 22.4 & 11.2 & 14.0 & 15.3 & 16.0 \\
\hline
\end{tabular}

Strohmayer (2001b) reported a $40 \mathrm{~Hz}$ QPO seen sometimes simultaneously with the more stable $67 \mathrm{~Hz}$ QPO (Morgan et al. 1997). QPOs have been observed with the following increasing frequencies 27-40-56-67 Hz (Belloni et al. 2001; van der Klis 2004). Some LF-QPOs varying between 1 and $15 \mathrm{~Hz}$ have been investigated intensively by Markwardt et al. (1999). Their properties seem to be different from the HF-QPOs, probably implying a different physical mechanism at work. We emphasize that it is not the scope of this paper to explain all the observed QPOs but just to explain or predict the HF-QPOs. LF-QPOs should be related to Lense-Thirring precession or to some other (magneto)hydrodynamical modes in the accretion disk.

Nevertheless, from the frequencies of the twin HF-QPOs, the constant angular pattern speed of the density wave is derived from our model by $v_{\mathrm{w}}=\Omega_{\mathrm{w}} / 2 \pi=v_{1} / 2=\nu_{2} / 3 \approx$ $56 \mathrm{~Hz}$. Putting this value of the gravitational field pattern speed into Table 1 we get the results shown in Table 2. There is a problem on selecting the relevant and meaningful frequencies in this table. Indeed, the spiral density wave travels outwards to several tens of Schwarzschild radii. According to Mao et al. (2008), the precise extension of the propagation of the wave with constant frequency and without attenuation depends on the accretion rate and on the viscosity (at least for their axisymmetric hydrodynamical modes). Assuming that the wave can travel only up to a radius $r_{\text {out }}$, for a black hole of mass $M_{\mathrm{BH}}$ and angular momentum $a_{\mathrm{BH}}$, the lowest excited frequency is

$v_{\text {low }}=\frac{\Omega_{\text {low }}}{2 \pi}=\frac{32310 \mathrm{~Hz}}{M_{\mathrm{BH}} / M_{\odot}} \frac{1}{a_{\mathrm{BH}} / R_{g}+\left(r_{\text {out }} / R_{g}\right)^{3 / 2}}$

To give some orders of magnitude, let's use $M_{\mathrm{BH}}=10 M_{\odot}$, $a_{\mathrm{BH}}=0, r_{\mathrm{out}}=30 R_{g}$, we find $\nu_{\text {low }}=19.7 \mathrm{~Hz}$. Which frequencies to select depends strongly on the spiral wave properties, how far they can propagate, which azimuthal numbers $m$ are excited, at which amplitude and so on. The detailed investigation is left for future work. We can only claim that the observed QPOs are retrieved by our model. How to select them remains quantitatively unclear.

Let's discuss however the predicted QPO frequencies. The first two strongest resonances for each azimuthal mode $m$ with the plus sign used in (3) match with good accuracy the frequencies observed in GRS $1915+105$. We find namely, in decreasing order, 168.0, 112.0, 56.0, 42.0 and $28.0 \mathrm{~Hz}$ for the most significant values. Some other resonances should also appear, but due to their higher order (higher integer $n$ ) and location farther away from the inner edge of the disk (thus an amplitude of the excitation decreasing with radius), they do not possess a significant growth rate relevant for the study presented here. Moreover, X-ray emission from the outer part of the disk is fainter, thus more difficult to detect. As a consequence, the parametric resonance induced by a spiral density wave passing through an accretion disk predicts the HF-QPOs as well as some LF-QPOs (as a byproduct) in a single unified picture. However, there is no way to predict the angular momentum of the black hole without some knowledge of its mass. We can derived a relation mass-spin to constrain the BH parameters, see next section. Nevertheless, it is worthwhile noting that Kato (2004) was lead to the conclusion that this microquasar is well described by a non rotating geometry indicating that the black hole angular momentum is weak, $a_{\mathrm{BH}} \ll R_{g}$ by fitting the HF-QPOs and several LF-QPOs with a resonant interaction between non linear oscillations and warp modes in the accretion disk. He found $a_{\mathrm{BH}}=0-0.15 R_{g}$.

Note also that the LF-QPOs $(27-41-56 \mathrm{~Hz})$ are more difficult to detect than the HF-QPOs $(113-165 \mathrm{~Hz})$ because they are located in regions of the accretion disk where emission is fainter. They correspond also to higher azimuthal modes ( $m=2,3$ compared to $m=1$ ). Assuming that the main wave possesses an $m=1$ structure, the amplitude of the LF-QPOs is expected to be small compared to those of the HF-QPOs. Furthermore, it seems that the presence of these features depend on the emission state of the black hole. We would then expect the accretion rate to have an influence on the efficiency of the parametric resonance occurring in the system. However, the constancy of the QPOs frequency strongly supports the fact the they are closely related to the black hole properties and independent of the flow in the surrounding accretion disk. A hydrodynamical general relativistic description is required to verify this assessment and to check the behaviour of the accretion rate on the presence of certain QPOs. This would be the continuation of the work begun by Mao et al. (2008).

\subsection{Mass-spin relation for BHCs}

Strictly speaking, at this stage, our model cannot estimate independently the angular momentum and the mass of the black hole. Nevertheless, applying it to some BHCs, we are able to give some constrain on their mass, assuming a nonrotating or a maximally rotating black hole.

Following the work done by Mao et al. (2008), we assume that the spiral density wave is launched close to the 


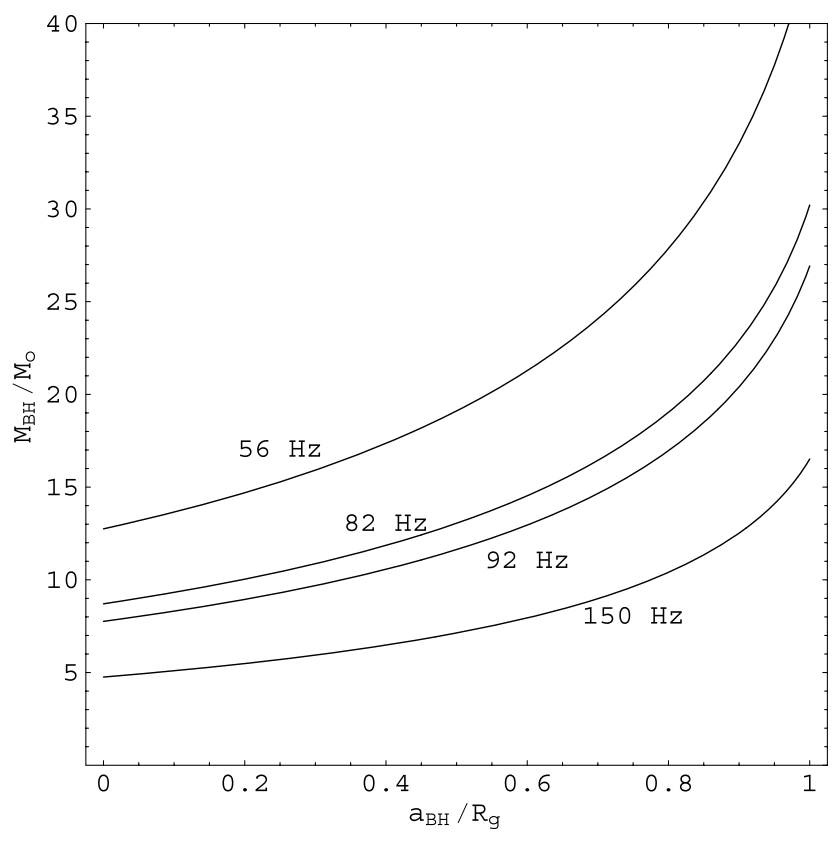

Fig. 1 The relation between mass $M_{\mathrm{BH}}$ (normalized to the mass of the Sun $M_{\odot}$ ) and angular momentum $a_{\mathrm{BH}}$ for different fundamental excitation frequencies corresponding to the four BHCs, namely, GRS 1915 + 105 with $56 \mathrm{~Hz}$, GRO J1655-40 with 150 Hz, XTE J1550-564 with $92 \mathrm{~Hz}$ and $\mathrm{H} 1743-322$ with $82 \mathrm{~Hz}$

ISCO, at the location where the radial epicyclic frequency is maximal, note this radius $r_{\max }$. The frequency of the spiral density wave is equal to the local orbital frequency at $r_{\max }$, thus the speed of the density perturbation pattern is $\Omega\left(r_{\max }, a_{\mathrm{BH}}\right)$. Knowing the fundamental frequency $\Omega_{\mathrm{w}}$ in the accretion disk for a given black hole, the resonance condition (2) puts constrains on the relation between mass and angular momentum by imposing $\Omega\left(r_{\max }, a_{\mathrm{BH}}\right)=\Omega_{\mathrm{w}}$.

This mass-angular momentum relation is shown in Fig. 1 for four BHCs (GRS $1915+105$, GRO J1655-40, XTE J1550-564, H1743-322) for which the fundamental frequency is known. If we assume a black hole weakly or mildly rotating, i.e. $a_{\mathrm{BH}} \leq 0.5 R_{g}$, we find that for GRS $1915+105$ its mass satisfies $13 M_{\odot} \leq M_{\mathrm{BH}} \leq 20 M_{\odot}$. The same model applied to other BHCs gives for GRO J165540 a mass $5 M_{\odot} \leq M_{\mathrm{BH}} \leq 7 M_{\odot}$ and for XTE J1550-564 a mass $8 M_{\odot} \leq M_{\mathrm{BH}} \leq 11 M_{\odot}$. This is consistent with other independent estimations of the $\mathrm{BH}$ mass. Finally for H1743-322, we found the following bounds, $9 M_{\odot} \leq$ $M_{\mathrm{BH}} \leq 13 M_{\odot}$.

\section{Conclusion}

In this paper, the consequences of an outgoing spiral density wave on the evolution of a test particle orbiting around a Kerr black hole have been explored. The twin peaks ratio around 3:2 for the kHz-QPOs is naturally explained by the parametric resonance model. The connection to lower frequency QPOs is also clearly demonstrated. It is an extension to accreting black hole for the model already suggested in neutron star and white dwarf binaries.

From the analysis of HF-QPOs, we are also able to constrain the mass and the spin of the hole as already suggested by Török et al. (2005). In the case of GRS $1915+105$, it appears that the $56 \mathrm{~Hz}$ feature in the Fourier time analysis is a fundamental frequency of the black hole from which the other QPOs can be derived. This feature should therefore be related to the intrinsic properties of the black, namely, its mass and its angular momentum. The way to associate $\Omega_{\mathrm{w}}$ with $a_{\mathrm{BH}}$ and $M_{\mathrm{BH}}$ remains unclear and needs further investigation but spiral density wave excitation close to the ISCO at nearly the maximum of the radial epicyclic frequency as suggested by Mao et al. (2008) is a interesting idea that needs further investigations.

Acknowledgements This work was partly supported by a grant from the French ANR MAGNET.

Open Access This article is distributed under the terms of the Creative Commons Attribution Noncommercial License which permits any noncommercial use, distribution, and reproduction in any medium, provided the original author(s) and source are credited.

\section{References}

Abramowicz, M.A., Kluźniak, W.: Astron. Astrophys. 374, L19 (2001) Abramowicz, M.A., Karas, V., Kluźniak, W., Lee, W.H., Rebusco, P.: Publ. Astron. Soc. Jpn. 55, 467 (2003)

Belloni, T., Méndez, M., Sánchez-Fernández, C.: Astron. Astrophys. 372, 551 (2001)

Bursa, M., Abramowicz, M.A., Karas, V., Kluźniak, W.: Astrophys. J. Lett. 617, L45 (2004)

Fender, R., Belloni, T.: Annu. Rev. Astron. Astrophys. 42, 317 (2004)

Kato, S.: Publ. Astron. Soc. Jpn. 56, L25 (2004)

Kluźniak, W., Abramowicz, M.A.: astro-ph/0203314 (2002)

Kluźniak, W., Abramowicz, M.A., Kato, S., Lee, W.H., Stergioulas, N.: Astrophys. J. Lett. 603, L89 (2004)

McClintock, J.E., Remillard, R.A.: astro-ph/0306213 (2003)

Mao, S.A., Psaltis, D., Milsom, J.A.: astro-ph/0805.0598 (2008)

Markwardt, C.B., Swank, J.H., Taam, R.E.: Astrophys. J. Lett. 513, L37 (1999)

Morgan, E.H., Remillard, R.A., Greiner, J.: Astrophys. J. 482, 993 (1997)

Morse, Feshbach: Methods of Theoretical Physics. McGraw-Hill, New-York (1953)

Pétri, J.: Astron. Astrophys. 439, L27 (2005a), paper I

Pétri, J.: Astron. Astrophys. 443, 777 (2005b), paper II

Pétri, J.: Astron. Astrophys. 439, 443 (2005c)

Pétri, J.: Astrophys. Space Sci. 302, 117 (2006)

Rebusco, P.: Publ. Astron. Soc. Jpn. 56, 553 (2004)

Remillard, R.A., McClintock, J.E.: Annu. Rev. Astron. Astrophys. 44, 49 (2006)

Rezzolla, L., Yoshida, S., Maccarone, T.J., Zanotti, O.: Mon. Not. R. Astron. Soc. 344, L37 (2003)

Schnittman, J.D., Bertschinger, E.: Astrophys. J. 606, 1098 (2004) 
Schnittman, J.D., Rezzolla, L.: arXiv:astro-ph/0506702 (2005)

Strohmayer, T.E.: Astrophys. J. Lett. 552, L49 (2001a)

Strohmayer, T.E.: Astrophys. J. Lett. 554, L169 (2001b)
Török, G., Abramowicz, M.A., Kluźniak, W., Stuchlík, Z.: Astron. Astrophys. 436, 1 (2005)

van der Klis, M.: astro-ph/0410551 (2004) 